\title{
Neurochemical effects of motor cortex stimulation in the periaqueductal gray during neuropathic pain
}

\author{
Emerson Magno de Andrade, MD, PhD, ${ }^{1,2}$ Raquel C. R. Martinez, PhD, ${ }^{2}$ Rosana L. Pagano, PhD, ${ }^{2}$ \\ Patricia S. S. Lopes, ${ }^{2}$ Aline V. V. Auada, PhD, ${ }^{3}$ Flavia V. Gouveia, PhD, ${ }^{2}$ Geiza F. Antunes, ${ }^{2}$ \\ Danielle V. Assis, ${ }^{2}$ Ivo Lebrun, PhD, ${ }^{3}$ and Erich T. Fonoff, MD, PhD ${ }^{1,2}$
}

1Department of Neurology, School of Medicine, University of São Paulo; '2Laboratory of Neuroscience, Hospital Sirio-Libanes; and ${ }^{3}$ Biochemistry and Biophysics Laboratory, Butantan Institute, University of São Paulo, São Paulo, Brazil

OBJECTIVE Motor cortex stimulation (MCS) is a neurosurgical technique used to treat patients with refractory neuropathic pain syndromes. MCS activates the periaqueductal gray (PAG) matter, which is one of the major centers of the descending pain inhibitory system. However, the neurochemical mechanisms in the PAG that underlie the analgesic effect of MCS have not yet been described. The main goal of this study was to investigate the neurochemical mechanisms involved in the analgesic effect induced by MCS in neuropathic pain. Specifically, we investigated the release of $\gamma$-aminobutyric acid (GABA), glycine, and glutamate in the PAG and performed pharmacological antagonism experiments to validate of our findings.

METHODS Male Wistar rats with surgically induced chronic constriction of the sciatic nerve, along with sham-operated rats and naive rats, were implanted with both unilateral transdural electrodes in the motor cortex and a microdialysis guide cannula in the PAG and subjected to MCS. The MCS was delivered in single 15-minute sessions. Neurotransmitter release was evaluated in the PAG before, during, and after MCS. Quantification of the neurotransmitters GABA, glycine, and glutamate was performed using a high-performance liquid chromatography system. The mechanical nociceptive threshold was evaluated initially, on the 14th day following the surgery, and during the MCS. In another group of neuropathic rats, once the analgesic effect after MCS was confirmed by the mechanical nociceptive test, rats were microinjected with saline or a glycine antagonist (strychnine), a GABA antagonist (bicuculline), or a combination of glycine and GABA antagonists (strychnine+bicuculline) and reevaluated for the mechanical nociceptive threshold during MCS.

RESULTS MCS reversed the hyperalgesia induced by peripheral neuropathy in the rats with chronic sciatic nerve constriction and induced a significant increase in the glycine and GABA levels in the PAG in comparison with the naive and sham-treated rats. The glutamate levels remained stable under all conditions. The antagonism of glycine, GABA, and the combination of glycine and GABA reversed the MCS-induced analgesia.

CONCLUSIONS These results suggest that the neurotransmitters glycine and GABA released in the PAG may be involved in the analgesia induced by cortical stimulation in animals with neuropathic pain. Further investigation of the mechanisms involved in MCS-induced analgesia may contribute to clinical improvements for the treatment of persistent neuropathic pain syndromes.

https://thejns.org/doi/abs/10.3171/2018.7.JNS173239

KEYWORDS motor cortex stimulation; neuropathic pain; periaqueductal gray; neurotransmitter; rats; peripheral nerve disease

$\mathrm{S}$ INCE the initial report by Tsubokawa et al. in $1991^{38}$ indicating that motor cortex stimulation (MCS) produces analgesia in patients with poststroke thalamic pain, MCS has gained notoriety for its effectiveness in the treatment of chronic neuropathic pain syndromes. ${ }^{8,10}$
MCS has since been used for the treatment of patients with chronic pain resistant to conventional pharmacological treatments, despite the lack of a complete understanding of the mechanisms underlying the analgesic effect of MCS. ${ }^{10,17}$ Pain syndromes treated with MCS include tri-

ABBREVIATIONS BL = baseline; $\mathrm{CCI}=$ chronic constriction injury; $\mathrm{GABA}=\gamma$-aminobutyric acid; $\mathrm{MCS}=$ motor cortex stimulation; $\mathrm{PAG}=$ periaqueductal gray; $\mathrm{PS}=$ poststimulation; RVM = rostral ventromedial medulla.

SUBMITTED January 2, 2018. ACCEPTED July 24, 2018

INCLUDE WHEN CITING Published online January 4, 2019; DOI: 10.3171/2018.7.JNS173239. 
geminal neuropathic pain, phantom limb pain, postherpetic neuralgia, poststroke pain, complex regional pain syndrome, pain secondary to multiple sclerosis, and posttraumatic brain and peripheral nerve injury pain. ${ }^{8,10,32}$

The periaqueductal gray (PAG) matter plays a key role in the descending pain inhibitory system, acting mainly through the rostral ventromedial medulla (RVM) to inhibit nociceptive neurons in the dorsal horn of the spinal cord. ${ }^{1,17,30}$ MCS elicits a substantial and selective antinociceptive effect by inhibiting thalamic sensory neurons and disinhibiting the neurons in PAG, with the involvement of the opioid system. ${ }^{7,18,30}$ The PAG receives inputs from several brain areas, such as the thalamus, hypothalamus, medulla oblongata, and spinal cord., ${ }^{1,18,27}$ The motor system appears to be connected largely to the PAG since the motor cortex projects to the ventral and ventrolateral parts of the PAG. ${ }^{1,11,27}$ Furthermore, experimental data have demonstrated that the motor cortex may be the most rostral neuroaxis structure that is responsible for pain modulation. $7,9,11,30$

Endogenous opioids and cannabinoids can regulate pain transmission by modulating the release of neurotransmitters in the PAG. ${ }^{15,20,21,34,43}$ The first report of amino acids playing an important role in modulating pain in the PAG was published by Sherman and Gebhart in 1974; ${ }^{35}$ these investigators observed that a nociceptive stimulus induces a significant reduction in glutamate levels in the PAG. Bearing in mind that glutamate is the main excitatory neurotransmitter and that $\gamma$-aminobutyric acid (GABA) and glycine act as the main inhibitory neurotransmitters, a dynamic characterization of amino acids is especially valuable for understanding the pain modulation system. ${ }^{6,13,15,31}$ Evidence is emerging that excitatory and inhibitory amino acids play important functional roles in the PAG and in other brain centers involved in pain modulation. ${ }^{13,15,20,25}$ Although there is both clinical and experimental evidence that these amino acids are involved in pain transmission, ${ }^{13,15,20,25,26,31,34}$ the mechanism underlying the analgesic effect of MCS on neurotransmitter release in the PAG remains unclear, and very little is known about the functions of the amino acids in the modulatory system of pain in the PAG.

This study was designed to investigate the neurochemical mechanisms involved in the analgesic effect induced by epidural MCS in neuropathic pain. Concentrations of GABA, glycine, and glutamate in the PAG of naive and sham-operated rats and rats with surgically induced neuropathy, all subjected to MCS, were measured and, to validate our findings, pharmacological antagonism was performed through PAG microinjections of antagonists of glycine, GABA, or a combination of glycine and GABA. The results of this study may contribute to the elucidation of the mechanisms of the analgesic effect of MCS, a phenomenon that is not fully understood.

\section{Methods \\ Study Design}

Two experiments were performed: assessment of amino acid levels in the PAG after MCS and determination of whether the analgesia induced by MCS was reversed by the microinjection of antagonists of the neurotransmitters glycine and GABA in the PAG (Fig. 1). Prior to experimentation and after a habituation period, the animals were randomly divided into control groups (naive or sham rats) or neuropathic rats. In the second experiment, just neuropathic rats were used. After randomization all animals underwent behavioral nociceptive testing before surgery, which provided baseline measurements that were subsequently used to assess homogeneity between groups. The day after nociceptive testing, animals in each group were treated as follows: 1) subjected to surgically induced chronic constriction injury (CCI) of the hind paw, which led to peripheral neuropathy (CCI group); 2) subjected to sham surgery (sham group); or 3) subjected to no surgical intervention in the hind paw (naive group). Seven days after the surgical procedures, all groups underwent in a single procedure the implantation of stimulation electrodes over the area of the motor cortex to be used for MCS and cannulas in the PAG. Implantation was stereotactically guided using predetermined coordinates. At the end of the experiments, the rats were euthanized by transcardial perfusion and the brains were removed.

In the first experiment, to assess the amino acid levels in the PAG after MCS, 14 days after the induction of peripheral neuropathy or sham surgery, animals were reevaluated with nociceptive testing before and during the MCS. The next day, the animals (CCI, sham, and naive group) were anesthetized, and the measurement of neurotransmitters was performed using the microdialysis cannulas in the PAG before, during, and 30 minutes after MCS. At the end of the experiment, after euthanasia and brain removal, histological slices of the brain were made and used to evaluate the targets of insertion of the microdialysis probe (Fig. 1A).

Following the assessment of amino acid levels, an additional experiment was performed to evaluate if the analgesia induced by the MCS could be reversed by microinjection of antagonists of glycine, GABA, or the combination of both antagonists. The CCI rats with epidural electrodes implanted over the motor cortex and guide cannula target to the PAG were reevaluated in the nociceptive test, before and during the MCS. Then, the rats were randomly assigned to microinjection of saline or a neurotransmitter antagonist (the glycine antagonist strychnine, the GABA antagonist bicuculline, or a combination of antagonists of both glycine and GABA [strychnine+bicuculline]). Thirty minutes after the microinjections, the rats were reevaluated with the nociceptive test during MCS. At the end of the experiment, after euthanasia and brain removal, histological slices of the brain were made and used for further analysis of the position of the cannula (Fig. 1B).

\section{Animals}

Sixty-three male Wistar rats weighing 170-190 g obtained from the animal facility of the University of São Paulo were used as experimental subjects. Animals were housed in polypropylene cages $(40 \times 34 \times 17 \mathrm{~cm})$ in groups of 3 rats per cage for at least 1 week before the beginning of the experimental procedures. The boxes, containing wood shavings, were maintained in a room with a con- 


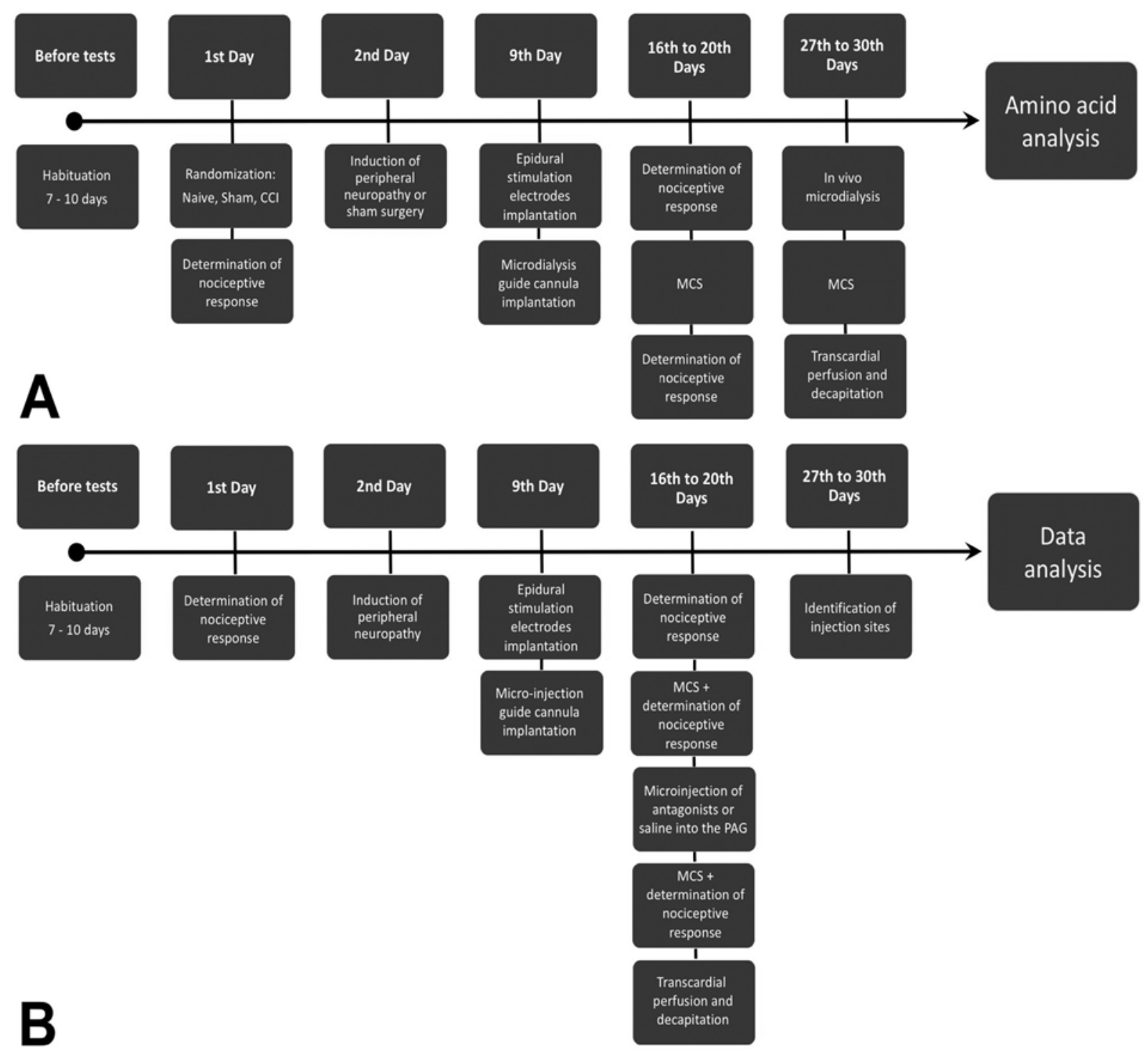

FIG. 1. A: Diagram of the study design for the amino acid evaluation. B: Diagram of the study design for the evaluation of the effects of the microinjection of antagonists of glycine, GABA, or a combination of both on the MCS.

trolled ambient temperature $\left(22^{\circ} \mathrm{C} \pm 2{ }^{\circ} \mathrm{C}\right)$ and a $12 / 12$ hour light/dark cycle, and the animals received water and rat chow pellets ad libitum. All experimental procedures were conducted in accordance with the Animal Research Reporting of In Vivo Experiments (ARRIVE) ${ }^{16}$ and the guidelines for the ethical use of animals in research involving pain and nociception. ${ }^{46}$ This study was approved by the Ethics Committee on the Use of Animals at Hospital Sírio-Libanês (protocol no. CEUA 2012/05) and the University of São Paulo Medical School (protocol no. CEUA 009/13).

\section{Induction of Neuropathic Pain}

For induction of neuropathic pain, peripheral neuropathy was induced through a CCI of the sciatic nerve performed by placing 4 loose ligatures around the nerve according to the method described by Bennett and $\mathrm{Xie}^{2}$ (additional details are provided in the Supplemental Material). In the animals in the sham group, the sciatic nerve was exposed, but no constriction was performed.

\section{Stimulation Electrode Implantation}

One week after the induction of peripheral neuropathy or sham surgery, the animals were deeply anesthetized by intramuscular administration of ketamine $(0.5 \mathrm{mg} / \mathrm{kg})$ and xylazine $2 \%(2.3 \mathrm{mg} / \mathrm{kg})$ with local anesthesia of the scalp with $2 \%$ lidocaine $(0.5 \mathrm{ml} / \mathrm{animal})$. Under stereotactic conditions, a pair of standard electrodes was implanted through 2 small trepanations localized over the left motor cortex in the cortical area functionally related to the right hind paw $(1.0 \mathrm{~mm}$ rostral and $1.5 \mathrm{~mm}$ caudal to the bregma and $1.5 \mathrm{~mm}$ lateral to midline), according to a previously established protocol. ${ }^{33}$ For additional information, see the Supplemental Material. 


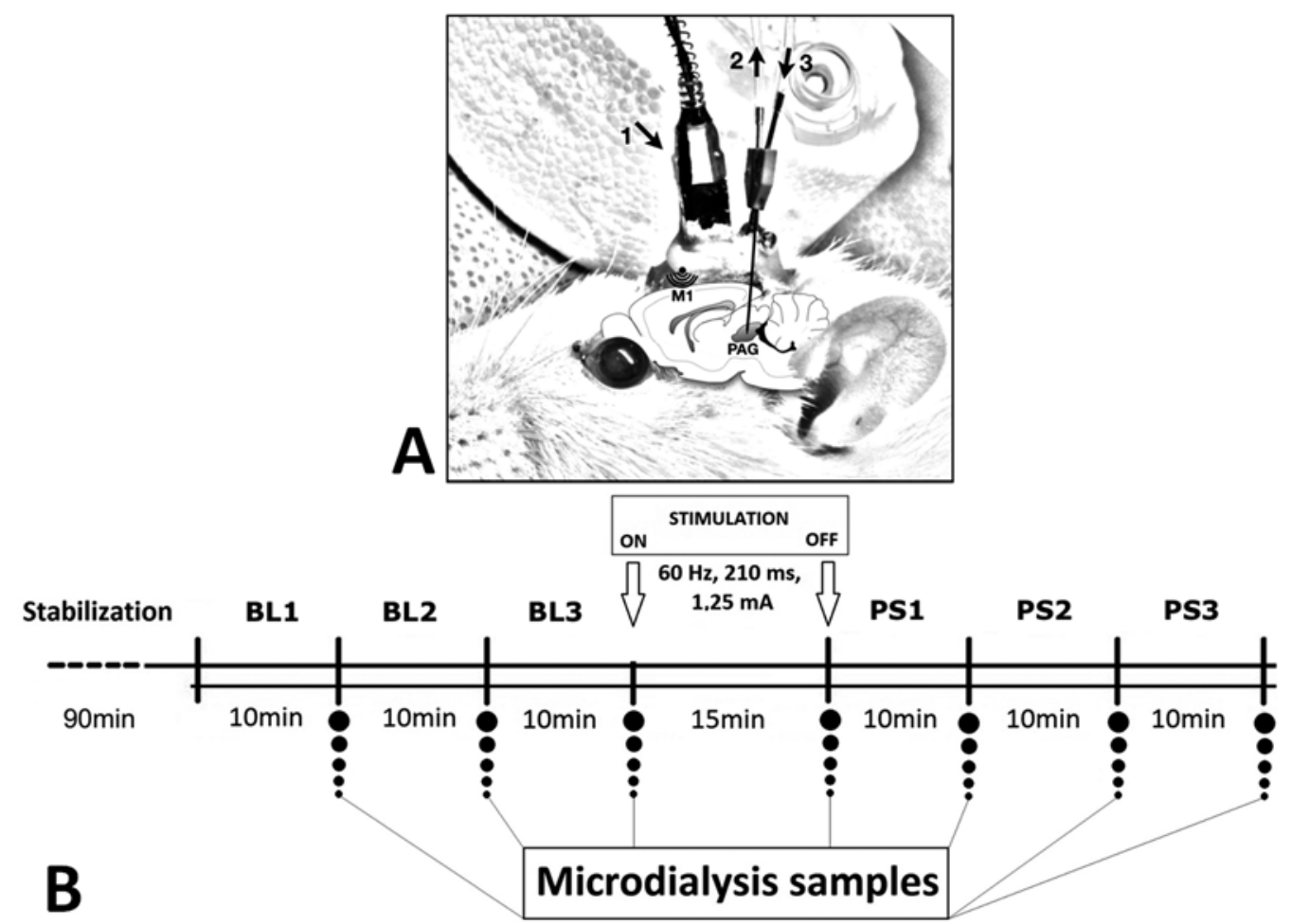

FIG. 2. Neurotransmitter collection. A: Photograph of a rat showing the guide cannula fixed to the skull and implanted in a caudal position in relation to the location of the stimulation electrodes, thus allowing the coupling of cables for stimulation and insertion of the microdialysis cannula appropriately and simultaneously. 1 = stimulation electrode; 2 = outlet conduit (dialysate); $3=$ inlet tube (perfusate); M1 = motor cortex. B: Schematic diagram of the motor cortex stimulation and collection of the microdialysis samples. PS = poststimulation.

\section{Microdialysis Guide Cannula Implantation}

For in vivo microdialysis, a special guide cannula and probe (CMA 12) were used. The microdialysis probe had an exposed membrane of $2 \mathrm{~mm}$ in length and $0.5 \mathrm{~mm}$ in diameter. The microdialysis guide cannula and the stimulation electrodes were concomitantly implanted under stereotactic conditions (for additional information, see the Supplemental Material). The microdialysis probe was targeted to the PAG by use of the following coordinates from the bregma according to the atlas: ${ }^{32}$ anteroposterior, $-7.8 \mathrm{~mm}$; mediolateral, $-0.7 \mathrm{~mm}$; and dorsoventral, -6.4 $\mathrm{mm}$. The implantation was performed in order to allow coupling of the cables for stimulation and appropriate and simultaneous insertion of the cannula microdialysis (Fig. 2A).

\section{Microinjection Guide Cannula Implantation}

During the same surgical procedure used for the electrode implantation, each animal was stereotactically implanted with unilateral stainless steel guide cannulas (17 $\mathrm{mm}, 24 \mathrm{G}$ ) targeted to the PAG according to the following coordinates from the bregma based on the atlas: ${ }^{32}$ anteroposterior, $-7.8 \mathrm{~mm}$; mediolateral, $-0.7 \mathrm{~mm}$; and dorsoventral, $-6.4 \mathrm{~mm}$. At the end of the surgery, fixation was performed with stainless steel screws and dental cement. A stainless steel wire was placed into the guide cannula to protect it from obstruction.

\section{Drugs}

Strychnine (Sigma Aldrich), an antagonist of glycine receptors, and bicuculline (Sigma Aldrich), an antagonist of GABA receptors, were used in the study. The strychnine and bicuculline were dissolved with artificial CSF (buffer solution) to concentrations of $2 \mathrm{mM}$ and $0.05 \mathrm{mM}$, respectively, and the animals were tested 30 minutes after the microinjection based on previously reported work. ${ }^{33}$

\section{Drug Microinjection}

For drug administration, the animals were microinjected with either saline, strychnine, bicuculline, or strychnine+bicuculline. The saline or antagonists were injected over a period of 2 minutes into the PAG $(0.2 \mu \mathrm{l} /$ min) using an infusion pump (Harvard Apparatus). For those animals administered strychnine+bicuculline, one injection was performed after the other. This procedure has already been used successfully by our group. ${ }^{23,24}$ For additional information, see the Supplemental Material.

\section{Determination of Mechanical Nociceptive Threshold}

The nociceptive threshold was determined using a pressure apparatus on the right hind paw (EEF-440, Insight). The test was carried out as follows: before surgery in all 3 groups (CCI, sham, and naive) to establish the baseline nociceptive threshold of each animal (initial measure- 
ment); on the 14th day following the surgery to confirm the neuropathic condition (final measurement); and after 15 minutes of MCS, while still under stimulation, to establish the effectiveness of MCS (MCS measurement). In the microinjected group, an additional measurement was evaluated 30 minutes after the microinjection of saline or the antagonists and while undergoing MCS. For additional information, see the Supplemental Material.

\section{Motor Cortex Stimulation}

After the surgical procedure for implanting the electrodes and microdialysis guide cannulas, the rats remained under observation for 7 days of recovery. One week after the surgical implantation, the MCS was delivered in a single 15-minute session in accordance with the parameters described previously $(1.0 \mathrm{~V}, 60 \mathrm{~Hz}$, and $210 \mu \mathrm{sec}$; Medtronic). ${ }^{9,29}$ Delivery according to these parameters allowed an increase of the pain threshold without interfering with the motor function of the animals. ${ }^{7,9,29}$ The rats were randomly divided into sham and stimulated groups.

\section{In Vivo Microdialysis}

Animals were anesthetized by intramuscular administration of ketamine $(0.5 \mathrm{mg} / \mathrm{kg})$ and xylazine $2 \%(2.3$ $\mathrm{mg} / \mathrm{kg}$ ), and the microdialysis stylet was replaced by the microdialysis probe. The probes were irrigated with artificial CSF at a rate of $2.0 \mu \mathrm{l} / \mathrm{min}$. After 90 minutes of stabilization, the dialysis material was collected in 7 different samples. Initially, 3 samples were collected every 10 minutes corresponding to the baseline (BL) (BL1, BL2, BL3). After collection of the basal samples, MCS was performed, using the parameters previously described, for 15 minutes. During the 15-minute stimulation period, one sample was collected (S), and during the poststimulation period (PS), 3 samples were collected every 10 minutes (PS1, PS2, PS3), as summarized in Fig. 2B. The dialysates were stored at $-80^{\circ} \mathrm{C}$ prior to the analysis.

\section{Amino Acid Analysis}

The dialysis samples were analyzed blindly using highperformance liquid chromatography (HPLC) (Shimadzu, Prominence UFLC). Quantification of the amino acids was performed as previously described. ${ }^{41}$ For additional information, see the Supplemental Material. The extracellular glutamate, glycine, and GABA levels in the dialysate were expressed as pigograms per liter.

\section{Histology}

At the end of the experiments, the animals were euthanized. In this procedure they were deeply anesthetized with a xylazine and ketamine solution and perfused through the left ventricle with $0.9 \%$ saline, followed by $4 \%$ paraformaldehyde dissolved in $0.1 \mathrm{M}$ phosphate buffer ( $\mathrm{pH}$ 7.4). The brains were then collected and cryoprotected in $30 \%$ sucrose for 48 hours at $4^{\circ} \mathrm{C}$. For analysis, the brains were sliced into coronal sections $(30 \mu \mathrm{m})$ on a freezing microtome.

\section{Sharing of Resources and Data}

The datasets generated and/or analyzed during the cur- rent study are available from the corresponding author upon reasonable request.

\section{Statistical Analysis}

The results are expressed as the mean \pm standard error of the mean (SEM). The data were analyzed with two-way repeated measures ANOVA or one-way ANOVA, followed by Tukey's post hoc test. In addition, Pearson correlations were performed. Statistical analysis was performed using the GraphPad Prism software, version 4.02. In all tests, $p$ $<0.05$ was considered statistically significant.

\section{Results \\ MCS Effect on the Nociceptive Threshold}

In the CCI rats, peripheral neuropathy promoted mechanical hyperalgesia compared with the control rats (interaction $\mathrm{F}_{(1,4)}=51.22 ; \mathrm{p}<0.05$ ) (Fig. 3). MCS significantly increased the nociceptive threshold in the CCI animals compared to the initial and final measurements between the groups $(\mathrm{p}<0.05)$. The nociceptive threshold returned to baseline when the paw pressure test was repeated 15 minutes after disconnection of cortical stimulation. There was no significant difference in results for the naive and sham animals (Fig. 3A).

\section{Flowcharts of the Animals Used in the Procedure}

For the microdialysis study, 33 animals were initially included in the study, and at the end, 18 rats had finished the experimental protocol: 5 naive, 5 sham, and 8 CCI rats (Fig. 3B). The rats that did not complete the study (rats that had lost the implant or were nonresponsive to the MCS) were euthanatized. The final data included 10 measurements (3 nociceptive threshold measurements and $7 \mathrm{mi}-$ crodialysis samples) from each of the 18 rats that completed the protocol.

For the microinjection study (Fig. 3C), $30 \mathrm{CCI}$ rats were initially included and 19 animals finished the experimental protocol: saline group $(n=5)$, strychnine group ( $=4)$, bicuculline group $(n=6)$, and strychnine+bicuculline group $(n=4)$. Only the data from those 19 rats were included in the analysis.

\section{Location of the Cannulas}

The locations of the microdialysis probes and microinjection guide cannulas were evaluated. We observed that the probes were located mainly through the lateral and dorsolateral PAG. In some cases, a small portion of the probes was partially located in the ventrolateral region of the PAG (Fig. 4A). The microinjection guide cannulas were targeted mainly to the lateral and dorsolateral columns of the PAG (Fig. 4B). The animals in which the probes were incorrectly located outside the PAG were excluded from further experimentation (Fig. 3B and C).

\section{MCS Effect on Glycine, GABA, and Glutamate Levels in the PAG}

Our results demonstrated an increase in the glycine levels during MCS, PS1, and PS3 in the CCI group compared to the naive and sham groups $\left(\mathrm{F}_{(2,24)}=96.99 ; \mathrm{p}<0.05\right)$ 

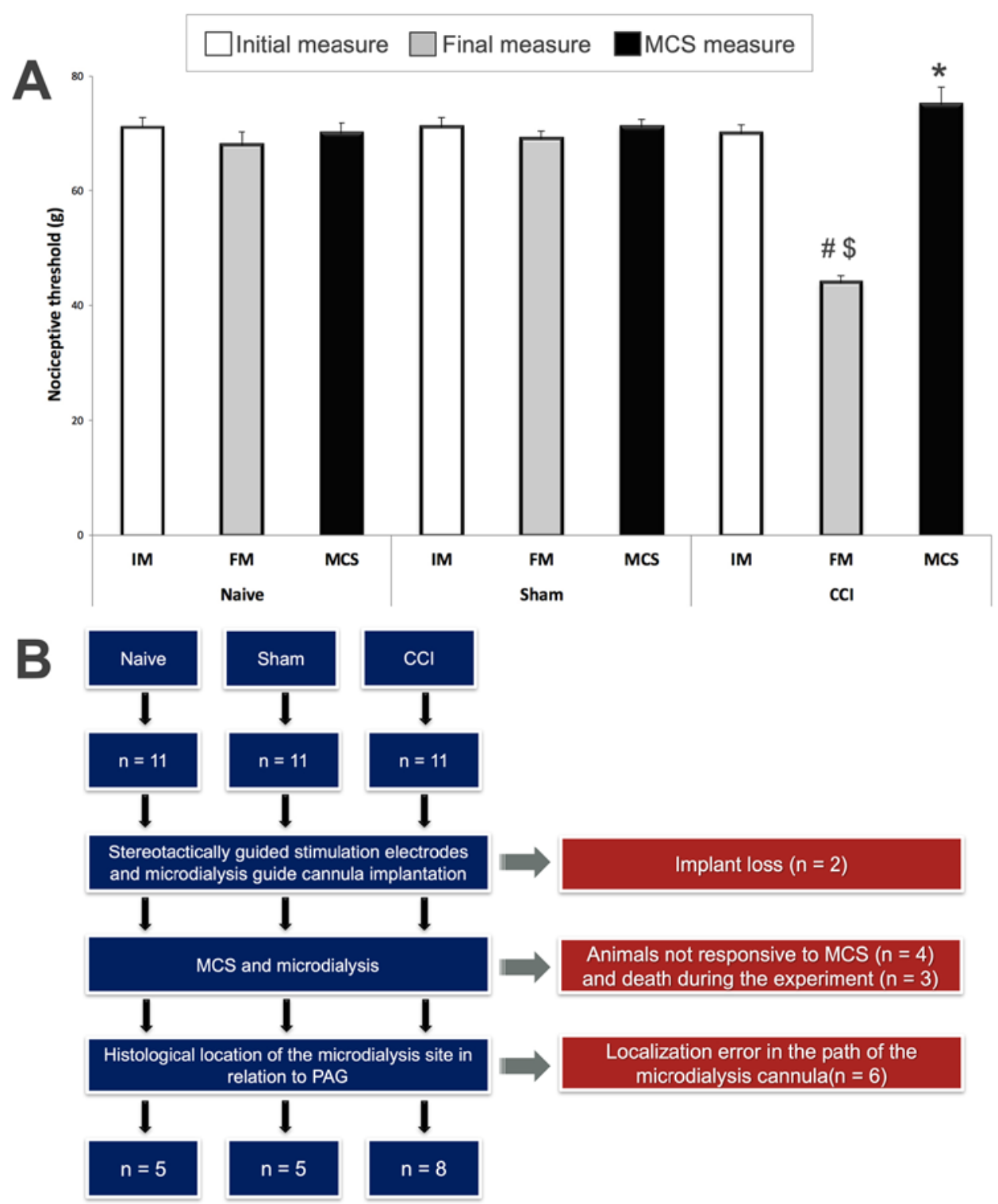

Induction of neuropathic pain $(n=30)$

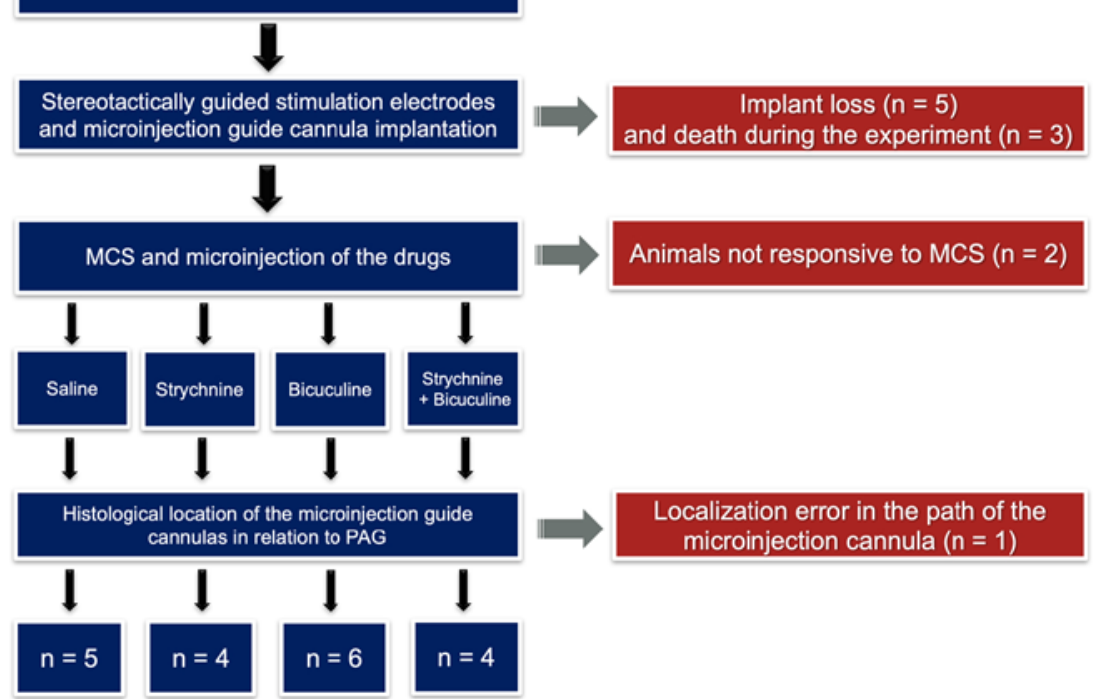

FIG. 3. A: MCS and nociceptive response. The nociceptive threshold was evaluated in the right hind paws of the rats with no surgical procedure (naive, $n=7$ ), with false surgery (sham, $n=11$ ), and with surgically induced peripheral neuropathy $(\mathrm{CCl}, \mathrm{n}=13)$. $\mathrm{CCl}$ or sham surgeries were performed in the right paws, and cortical electrodes were implanted in the left hemispheres of all animals. The nociceptive test was performed 1) before surgery (initial measurement [IM]), 2) 14 days after surgery (final measurement 1 [FM]), and 3) during MCS. \#p < 0.05 compared to IM; ${ }^{*} p<0.05$ compared to FM; $\$ p<0.05$ compared to all other groups. MCS did not affect nociceptive threshold in naive and sham animals, while $\mathrm{CCl}$ animals showed a decrease in the nociceptive threshold, which was readily reversed by MCS. B and C: Flowcharts of animals used in the 2 experiments, the microdialysis study (B) and the microinjection study $(\mathrm{C})$. Figure is available in color online only. 


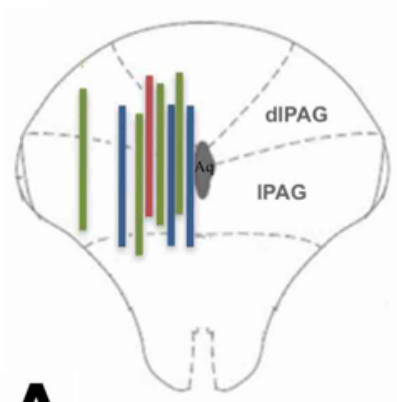

A

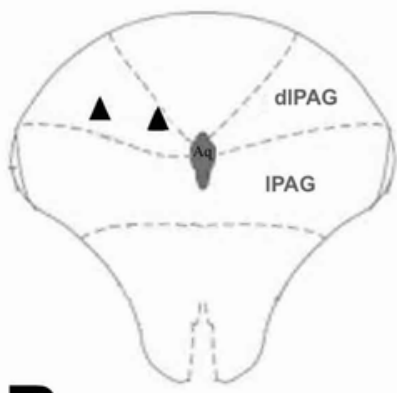

Bregma $-6.48 \mathrm{~mm}$

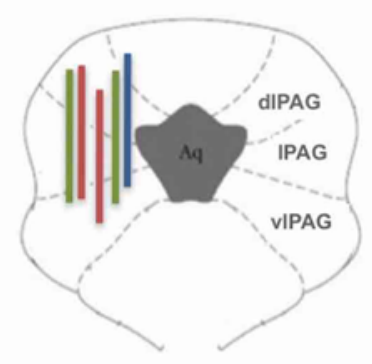

Bregma -7.44 mm

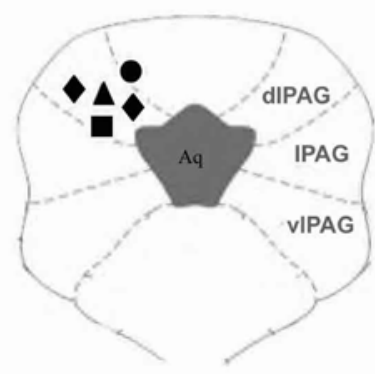

Bregma -7.44 mm

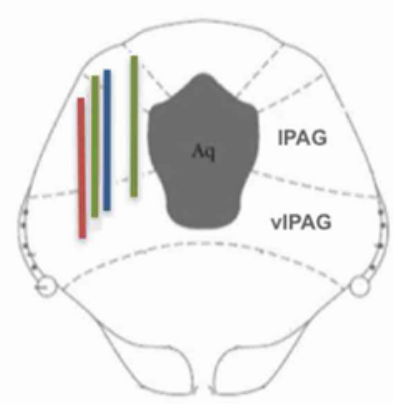

Bregma -7.92 mm

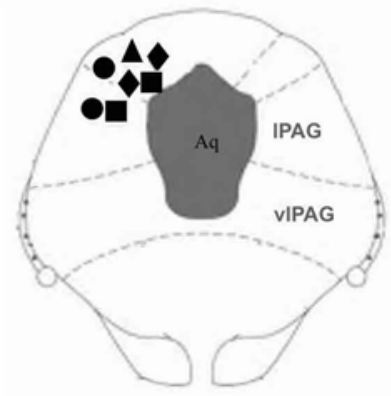

Bregma -7.92 mm
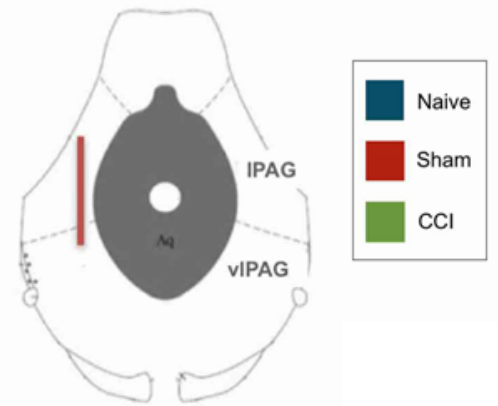

Bregma $-8.40 \mathrm{~mm}$
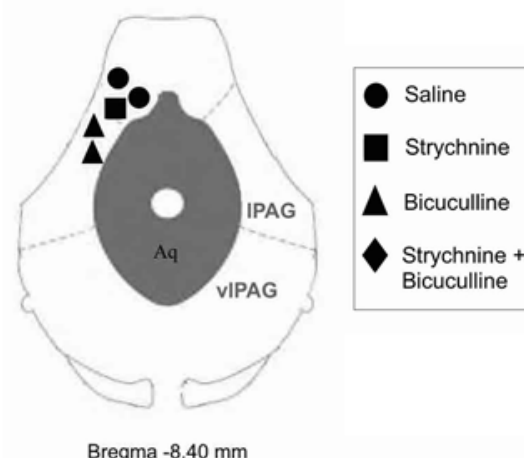

FIG. 4. Location of the microdialysis probes within the PAG. Histological localization of the PAG microdialysis sites in the rat coronal sections, redrawn from the Paxinos and Watson atlas (1998) ${ }^{32}$ at 4 representative rostrocaudal levels of the PAG, considering the microdialysis cannula $(\mathbf{A})$ and microinjection cannula $(B)$. Most of the microdialysis sites were located in the dorsal and lateral PAG. Figure is available in color online only.

(Fig. 5A). In addition, the CCI group showed an increase in the GABA levels during MCS compared to the naive and sham groups $\left(\mathrm{F}_{(2.24)}=106.65 ; \mathrm{p}<0.05\right)($ Fig. $5 \mathrm{~B})$, while glutamate levels in the PAG remained stable over all conditions in the 3 groups $\left(\mathrm{F}_{(2,2)}=0.19 ; \mathrm{p}>0.05\right)$ (Fig. 5C).

Pearson correlations were performed to analyze the nociceptive response of individual CCI rats to MCS and the changes in the baseline of GABA, glycine, and glutamate. There was a positive correlation for GABA $\left(r^{2}=0.49\right)$ and glycine $\left(r^{2}=0.27\right)$ and no correlation for glutamate $\left(r^{2}=\right.$ $0.00)$. Specifically, the most important observation was that animals with higher increases in the pain threshold after MCS had higher amounts of GABA released.

\section{MCS Effect After Microinjection of Saline or Antagonists of Glycine, GABA, or Glycine and GABA}

The nociceptive threshold evaluated during MCS was reduced in rats microinjected with strychnine, bicuculline, or strychnine+bicuculline compared with rats microinjected with saline $\left(\mathrm{F}_{(1,3)}=101.47 ; \mathrm{p}<0.05\right)$ (Fig. 6A). These data showed that microinjection of the antagonists of glycine, GABA, or a combination of both reversed the analgesic effect induced by the MCS.

All CCI rats were evaluated before and after surgical neuropathic induction. The surgically induced CCI promoted hyperalgesia compared with initial measurement (IM) and MCS significantly increased the nociceptive threshold: saline $\left(\mathrm{F}_{(13)}=238.84 ; \mathrm{p}<0.05\right)$, strychnine $\left(\mathrm{F}_{(1,3)}=516.08 ; \mathrm{p}<0.05\right)$, bicuculline $\left(\mathrm{F}_{(1,3)}=549.94 ; \mathrm{p}\right.$
$<0.05)$, and strychnine+bicuculline $\left(\mathrm{F}_{(1,3)}=91.79 ; \mathrm{p}<\right.$ $0.05)$. The microinjection of strychnine, bicuculline, or strychnine+bicuculline significantly reduced the nociceptive thresholds in comparison with MCS only, as can be seen in Fig. 6B-E.

\section{Discussion}

In this study, we investigated the analgesic therapeutic mechanisms of MCS using in vivo microdialysis and pharmacological microinjections focusing on the role of neurotransmitter amino acids in the PAG. Our results suggest that glycine and GABA within the PAG contribute to the antinociceptive effect of MCS. To our knowledge, this is the first study to evaluate MCS-induced analgesia based on the release and pharmacological antagonism of those amino acids using in vivo microdialysis and microinjection techniques. Initially we demonstrated that the experimental model was adequate by showing that MCS induced analgesia in CCI rats, as observed by the reversion of mechanical hyperalgesia, and that this effect ceased when stimulation was switched off, a finding that is in accordance with those of previous studies of MCS in experimental models ${ }^{7,11,29,30}$ and chronic pain patients. ${ }^{8,10}$ These observations reinforce the hypothesis that the primary motor cortex is one of the most rostral structures in the neuroaxis related to the pain modulating system and that transdural stimulation induces analgesia in neuropathic pain conditions. The neuroanatomical substrate for this analgesic effect could be a direct connection between the 

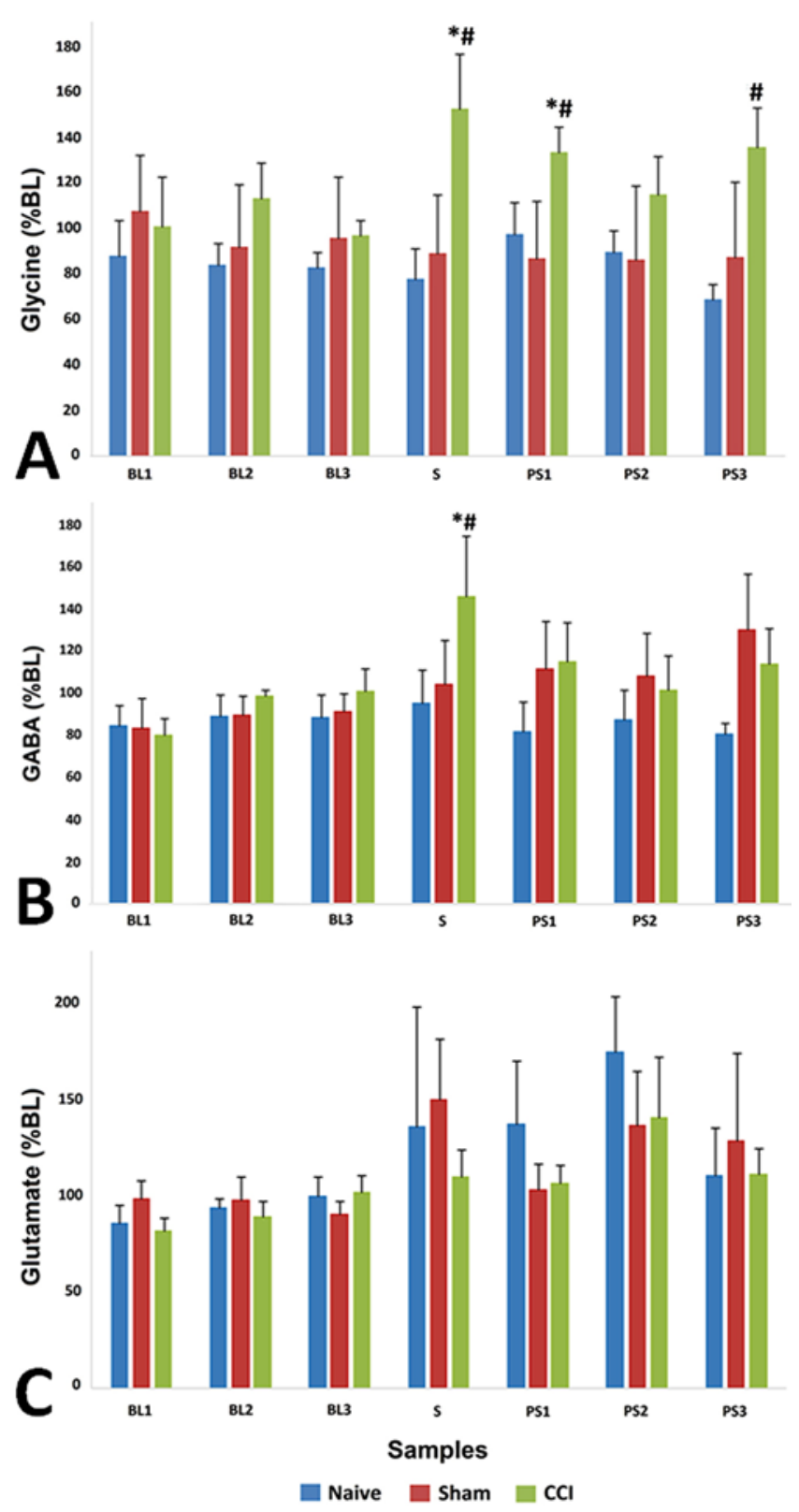

FIG. 5. Amino acid levels at baseline (BL1-3), MCS (S), and after stimulation (PS1-3) for the naive $(n=5)$, sham $(n=5)$, and $C C l$ groups $(n=$ 8); relative levels of glycine (A), GABA (B), and glutamate (C) according to the microdialysis sampling in the PAG. \#p $<0.05$ compared to the sham group; ${ }^{*} p<0.05$ compared to the naive group. Figure is available in color online only.

motor cortex and PAG, $1,7,17,27,30$ as illustrated in Fig. 7. The MCS-induced analgesia activates the PAG, which in turn activates the descending pain inhibitory pathways mainly through the RVM, which projects directly into the spinal cord dorsal horn. , $29,30,41^{2}$

However, in our study, unlike previous studies, $7,29,30,41$ we did not observe a marked modulator effect of MCS in naive rats. One possible explanation for this apparent inconsistency is that the control animals in the present study could not be considered completely naive. Although these animals had not undergone neuropathic pain induction, they did receive the microdialysis cannula implantation within the PAG, which according to our understanding is highly involved in the pain control mechanism.

Electrophysiological and neuroimaging studies suggest that the PAG plays a key role in the analgesia induced by epidural MCS. . $^{1729,30,41}$ The PAG presents distinct and opposing functions in the analgesic response since the naloxone opioid antagonist elevates the nociceptive threshold induced by ventrolateral PAG (vlPAG) stimulation but not by dorsolateral PAG (dlPAG) stimulation. The systems formed by these portions of the PAG should work through interactions between the PAG columns and their descendent systems. ${ }^{18,28}$ The existence of a cannabinergic painmodulatory mechanism within the dorsal and lateral PAG that is regulated by GABA, glycine, and glutamate similarly to pain-modulatory mechanisms regulated by opioids has been proposed. ${ }^{43}$

Since its early development in $1966,{ }^{3}$ microdialysis has become a valuable tool for measuring extracellular substances in different regions of the central nervous system. ${ }^{3,19-21,34}$ Despite its usefulness, there are several limitations to the microdialysis technique. During diffusion through the extracellular space, metabolism of the receptors and degradation of the neurotransmitters can modify the amounts of the components that are collected through the dialysate. ${ }^{3,19}$ Furthermore, depending on the area to be analyzed, the microdialysis cannula size, membrane properties, and tissue damage during cannula insertion may influence the results obtained and complicate data interpretation. ${ }^{3,19}$

Previous microdialysis studies have shown that glutamate is present at high basal concentrations in the spinal cord and in brainstem-related nuclei such as the PAG and RVM. ${ }^{20,34}$ In addition, differentially labeled receptors for glutamate are present at high levels in the PAG. ${ }^{6,15}$ Variations in the extracellular levels and in the distributions of all subtypes of glutamate binding sites in the PAG indicate the role of glutamate in pain modulation. ${ }^{6}$ Moreover, microinjection of excitatory amino acids into the PAG activates the descending pain inhibitory pathways. ${ }^{15}$ In this study, we found that glutamate release remained unchanged in the PAG microdialysates after MCS, even though glutamate was present at basal levels as previously reported..$^{20,34}$ This finding may be related to the PAG region analyzed, considering that the analgesic effect of glutamate is commonly described in vIPAG and that our microdialysis targets analyzed here were dorsomedial PAG, dlPAG, lateral PAG, and vlPAG. It is well known that in neuropathic pain models, glutamatergic neurotransmission in the vlPAG may be crucial to induce a persistent reduction in the descending pain inhibitory system, which results in chronic neuropathic pain. ${ }^{13}$ Additionally, our results also suggest that glutaminergic neurotransmission in the PAG may not be critical for MCS-induced analgesia.

Glycine may exert its inhibitory function through activation of specific postsynaptic receptors (GlyR) and an excitatory effect by acting as a coagonist of glutamate on the activation of $N$-methyl-D-aspartate (NMDA) receptors. ${ }^{5,25}$ GlyRs are found mainly in dlPAG. ${ }^{5,26}$ Additionally, a common vesicular transporter for both glycine and GABA, 

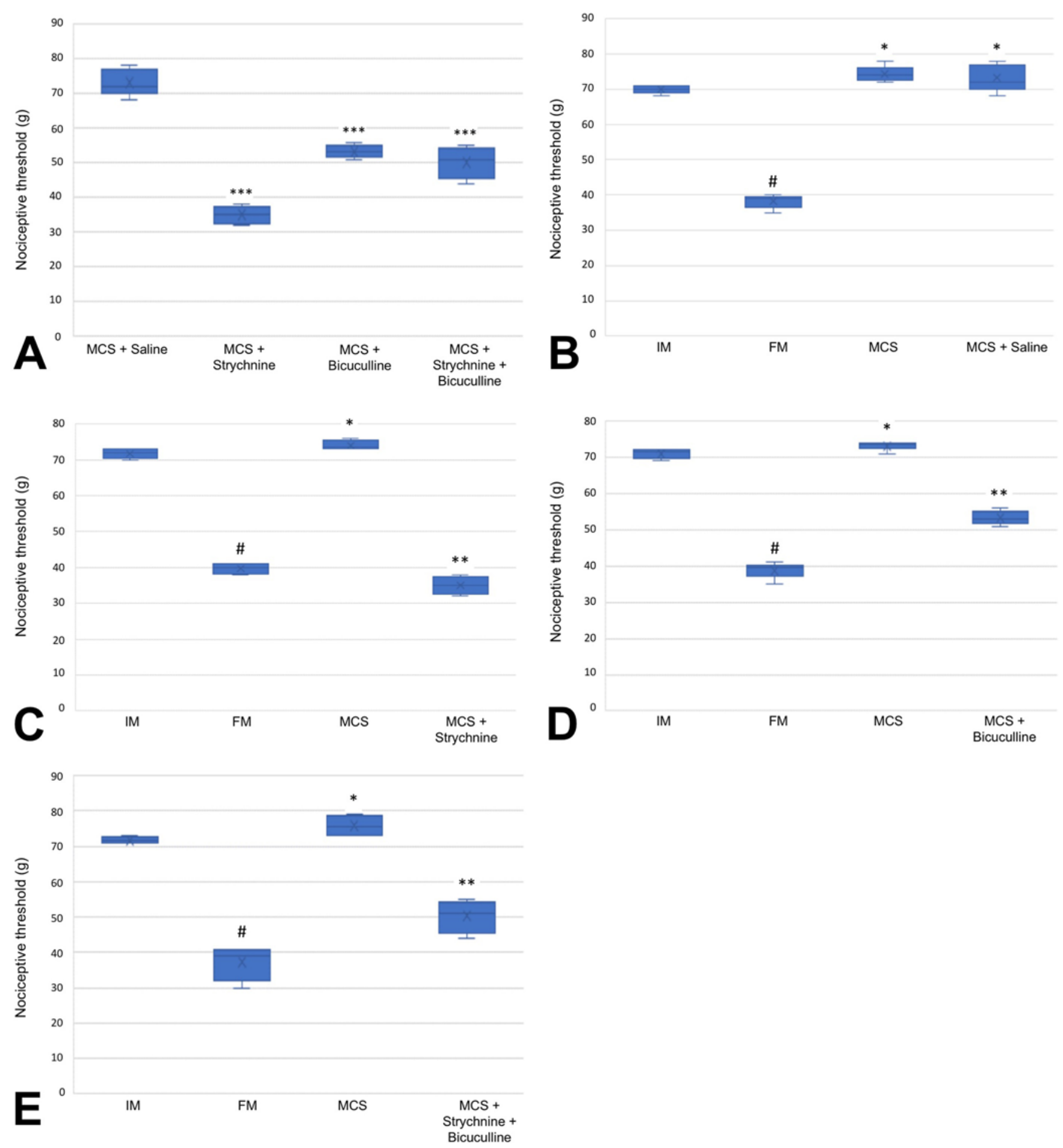

FIG. 6. (A) The nociceptive threshold data after intra-PAG microinjection. The evaluation was performed 30 minutes after the administration of saline, strychnine, bicuculline, or strychnine+bicuculline during MCS. For data from individual groups, the nociceptive threshold was evaluated in the right hind paws before the surgical manipulation (IM), after the induction of neuropathic pain (FM), and during the motor cortex stimulation (MCS). Then 30 minutes after the microinjection of $(B)$ saline $(n=5),(C)$ strychnine $(n=4)$, (D) bicuculline $(n=6)$, or $(E)$ strychnine+bicuculline $(n=4)$ into the PAG, $C C I$ rats were reevaluated during MCS. \#p $<0.05$ in comparison with IM; ${ }^{*} p<0.05$ in comparison with FM; ${ }^{* *} p<0.05$ in comparison with MCS; ${ }^{* * *} p<0.05$ in comparison with the saline group. Figure is available in color online only. 


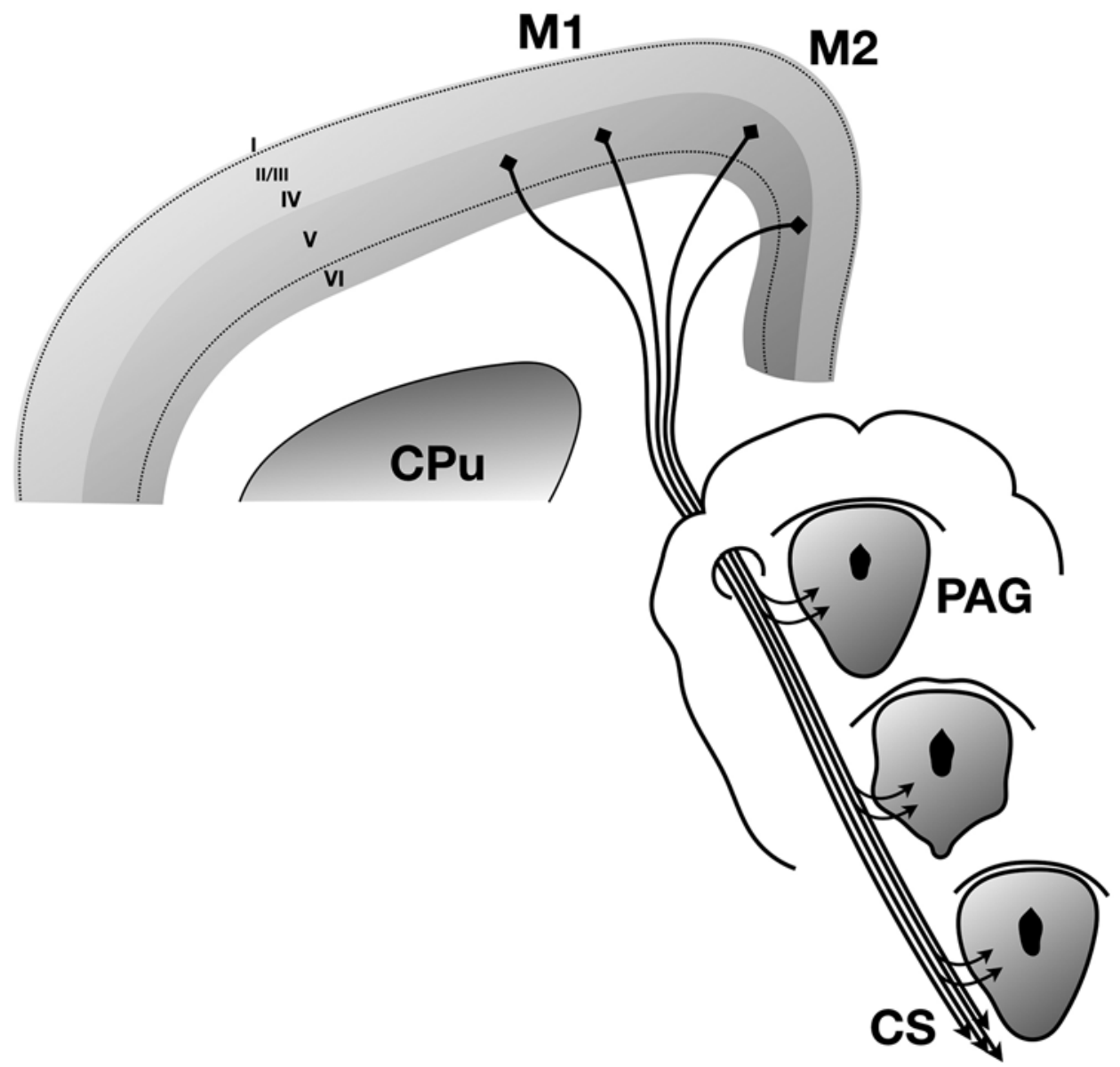

FIG. 7. Illustration showing the direct projections of the motor cortex to the ipsilateral PAG in the rat brain. $C P u=$ caudate-putamen complex; CS = corticospinal tract; M1 = primary motor cortex; M2 = secondary motor cortex.

which would be responsible for their costorage in the same synaptic vesicle and subsequent corelease at mixed synapses of these inhibitory neurotransmitters, has been proposed. It has been hypothesized that GABA and glycine are released by distinct populations of interneurons and not jointly in the PAG. ${ }^{20}$ Glycine plays a key role in pain modulation at supraspinal levels. ${ }^{25}$ Data obtained from in situ hybridization and immunoreactivity studies indicate that glycine seems to play an important role in pain processing in the PAG. ${ }^{5,31,33}$ In vivo microdialysis studies have shown that inflammatory pain markedly increases the glycine and glutamate levels and reduces GABA in the PAG. ${ }^{31,39}$ However, microinjection of glycine in the dlPAG induces antinociception in rats, and this effect is anatomically specific to dlPAG. ${ }^{25}$ Choi et al. ${ }^{5}$ reported that activation of presynaptic GlyR enhances glutamate release onto PAG neurons and therefore provides an antinociceptive function that is mediated by the PAG. However, glycine has a dual role in pain control; low doses of glycine into the dorsal PAG produce hyperalgesia, while high doses produce antinociception, suggesting that in the dorsal PAG, glycine produces facilitation or inhibition of nociception. ${ }^{26}$ In the present study, we observed an increase in glycine release during and after MCS and that the MCSinduced analgesia effect was reversed by the microinjec- tion of strychnine, an antagonist of glycine receptors, suggesting that glycine may be involved in the antinociceptive effect induced by MCS. Some studies have demonstrated that activation of glycine receptors increases the excitability of PAG neurons by enhancing excitatory glutamatergic transmission, ${ }^{25}$ however, we did not observe an increase in glutamate release during MCS, suggesting that glycine may be involved in MCS-induced analgesia and that this involvement is probably independent of glutamate action.

The amino acid GABA plays an important role in pain regulation in the PAG. GABA interneurons are present throughout the rostrocaudal extension of the PAG and may account for approximately $36 \%$ of neuronal populations in the vlPAG and dlPAG columns. ${ }^{34}$ At least two categories of GABAergic interneurons may exist in the PAG. The first group generates a tonic control of projecting neurons, and the second group acts on those GABAergic neurons and, in turn, maintains projecting neurons under tonic inhibition.? GABAergic terminals comprise almost $40 \%$ of all axon terminals in the vlPAG, and approximately $50 \%$ of PAG cells that are retrogradely labeled in the RVM have GABA-immunoreactive synaptic inputs to their soma and proximal dendrites. ${ }^{33,34,42,44}$ In the present study, we observed an increase in GABA release during MCS, a positive correlation between the MCS effect and GABA 
released, and the reversion of the analgesic effect after GABA receptor antagonism, emphasizing that GABA is essential for the modulation of analgesia, as previously described. ${ }^{36,40}$

Regarding the MCS effect, Pagano et al. showed that MCS enhanced neuronal activation and decreased GABA in the PAG, suggesting that MCS induced inhibition of GABAergic interneurons, consequently activating the descending analgesic pathway leading to the antinociceptive effect. ${ }^{30}$ However, the decrease in glutamic acid decarboxylase (GAD) expression that was previously shown ${ }^{30}$ was observed in naive animals, without neuropathic pain. In the present data, we observed an increase in the GABA levels only in the CCI animals, whereas the naive and sham rats did not show any change in GABA levels during stimulation. The discrepancy between the studies could have been attributable to differences due to plastic changes during the induction of neuropathic pain in the nociceptive circuitry, the time course of the evaluation, or the technical approach to the experimentation. Neuropathic pain is characterized by molecular and biochemical neuroplasticity that could occur in the ascending and descending pathways. ${ }^{4}$ Considering the time course of evaluating histological sections, Pagano et al. ${ }^{30}$ have chosen to evaluate GAD expression after 1 hour based on the peaks of expression of transcription factor proteins. ${ }^{12}$ On the other hand, microdialysis enables immediate and local sampling and collection of GABA from the interstitial space..$^{19,45}$ With regard to the technical approach, although both studies focused on cerebral chemical transmission, there is a difference in the measurement methods required for immunostaining for GABA versus microdialysis. Immunostaining markers label GABAergic neurons, including different intracellular pools, the cytoplasmic and vesicular GABA. ${ }^{42,44}$ Staining GAD in the brain allows evaluation of the GAD inactive reservoir from the cell bodies that could be activated when additional GABA synthesis is required. ${ }^{14,22}$ An advantage of using microdialysis is that this practical method evaluates dynamic changes in the extracellular space that offers reliable measurements of GABA influenced by GABAergic afferents. ${ }^{19,37}$ The present findings illustrate the complexity of the local and expanded network of nociception modulating transmitters and the key role of microdialysis in evaluating this neurotransmission process.

Regarding the limitations of the present study, one should consider that the PAG is a highly complex structure with subdivisions that exhibit different and opposing functions, as demonstrated by both electrical and neurochemical studies; moreover, the same neurotransmitters, such as glycine and glutamate, may produce diverse responses depending on the PAG regions where they operate. ${ }^{21,25,26,31} \mathrm{In}$ addition, the neurotransmitter collections were performed with anesthetized animals, and the anesthetization may have interfered with the release of the neurotransmitters, as previously demonstrated..$^{26}$ Our results suggest that the amino acids glycine and GABA that are released in the PAG may be involved in the analgesia induced by cortical stimulation in animals with neuropathic pain. Due to technical labeling limitations, we were not able to determine if the MCS induces morphological changes in the neurons. Additionally, the mechanism underlying the increase in the GABA and glycine levels induced by MCS is not completely clear, and more studies will be necessary to clarify the possible relationship between MCS-induced analgesia in neuropathic pain models and the GABA and glycine modifications in the PAG.

\section{Conclusions}

A comprehensive understanding of the therapeutic mechanism underlying the analgesic effect of MCS and the role of glycine and GABA is of great interest because it will help us to understand the modulation of pain transmission and may provide us with new treatment options for chronic pain conditions.

\section{Acknowledgments}

The authors are grateful to Professor José Pinhata Otoch and Mrs. Junko Takano Osaka of the Medical Investigation Laboratory (LIM 26), Department of Surgery Technique School of Medicine, University of São Paulo, Brazil. The authors thank the research assistants and staff of Hospital Sírio-Libanês (HSL).

R.C.R.M. and R.L.P. received FAPESP grants (11/08575-7 and $15 / 26079-8$, respectively) from the government of Brazil.

\section{References}

1. Beitz AJ: The organization of afferent projections to the midbrain periaqueductal gray of the rat. Neuroscience 7:133-159, 1982

2. Bennett GJ, Xie YK: A peripheral mononeuropathy in rat that produces disorders of pain sensation like those seen in man. Pain 33:87-107, 1988

3. Bito L, Davson H, Levin E, Murray M, Snider N: The concentrations of free amino acids and other electrolytes in cerebrospinal fluid, in vivo dialysate of brain, and blood plasma of the dog. J Neurochem 13:1057-1067, 1966

4. Boadas-Vaello P, Castany S, Homs J, Álvarez-Pérez B, Deulofeu M, Verdú E: Neuroplasticity of ascending and descending pathways after somatosensory system injury: reviewing knowledge to identify neuropathic pain therapeutic targets. Spinal Cord 54:330-340, 2016

5. Choi KH, Nakamura M, Jang IS: Presynaptic glycine receptors increase GABAergic neurotransmission in rat periaqueductal gray neurons. Neural Plast 2013:954302, 2013

6. de Novellis V, Marabese I, Palazzo E, Rossi F, Berrino L, Rodella L, et al: Group I metabotropic glutamate receptors modulate glutamate and $\gamma$-aminobutyric acid release in the periaqueductal grey of rats. Eur J Pharmacol 462:73-81, 2003

7. Fonoff ET, Dale CS, Pagano RL, Paccola CC, Ballester G, Teixeira MJ, et al: Antinociception induced by epidural motor cortex stimulation in naive conscious rats is mediated by the opioid system. Behav Brain Res 196:63-70, 2009

8. Fonoff ET, Hamani C, Ciampi de Andrade D, Yeng LT, Marcolin MA, Jacobsen Teixeira M: Pain relief and functional recovery in patients with complex regional pain syndrome after motor cortex stimulation. Stereotact Funct Neurosurg 89:167-172, 2011

9. Fonoff ET, Pereira JF Jr, Camargo LV, Dale CS, Pagano RL, Ballester G, et al: Functional mapping of the motor cortex of the rat using transdural electrical stimulation. Behav Brain Res 202:138-141, 2009

10. Fontaine D, Hamani C, Lozano A: Efficacy and safety of motor cortex stimulation for chronic neuropathic pain: critical review of the literature. J Neurosurg 110:251-256, 2009

11. França NRM, Toniolo EF, Franciosi AC, Alves AS, de Andrade DC, Fonoff ET, et al: Antinociception induced 
by motor cortex stimulation: somatotopy of behavioral response and profile of neuronal activation. Behav Brain Res 250:211-221, 2013

12. Herdegen T, Leah JD: Inducible and constitutive transcription factors in the mammalian nervous system: control of gene expression by Jun, Fos and Krox, and CREB/ATF proteins. Brain Res Brain Res Rev 28:370-490, 1998

13. Ho YC, Cheng JK, Chiou LC: Hypofunction of glutamatergic neurotransmission in the periaqueductal gray contributes to nerve-injury-induced neuropathic pain. J Neurosci 33:78257836, 2013

14. Itoh M, Uchimura H: Regional differences in cofactor saturation of glutamate decarboxylase (GAD) in discrete brain nuclei of the rat. Effect of repeated administration of haloperidol on GAD activity in the substantia nigra. Neurochem Res 6:1283-1289, 1981

15. Jacquet YF: The NMDA receptor: central role in pain inhibition in rat periaqueductal gray. Eur J Pharmacol 154:271276, 1988

16. Kilkenny C, Browne WJ, Cuthill IC, Emerson M, Altman DG: Improving bioscience research reporting: the ARRIVE guidelines for reporting animal research. PLoS Biol 8:e1000412, 2010

17. Kim J, Ryu SB, Lee SE, Shin J, Jung HH, Kim SJ, et al: Motor cortex stimulation and neuropathic pain: how does motor cortex stimulation affect pain-signaling pathways? J Neurosurg 124:866-876, 2016

18. Linnman C, Moulton EA, Barmettler G, Becerra L, Borsook D: Neuroimaging of the periaqueductal gray: state of the field. Neuroimage 60:505-522, 2012

19. Lopez WOC, Fonoff ET, Hamani C, Tierney TS, Alho E, Ghilardi MG dos S, et al: Optimizing microdialysis for deep brain stimulation. Front Biosci (Elite Ed) 8:299-310, 2016

20. Maione S, Marabese I, Oliva P, de Novellis V, Stella L, Rossi $F$, et al: Periaqueductal gray matter glutamate and GABA decrease following subcutaneous formalin injection in rat. Neuroreport 10:1403-1407, 1999

21. Maione S, Marabese I, Rossi F, Berrino L, Palazzo E, Trabace L: Effects of persistent nociception on periaqueductal gray glycine release. Neuroscience 97:311-316, 2000

22. Martin DL, Martin SB, Wu SJ, Espina N: Regulatory properties of brain glutamate decarboxylase (GAD): the apoenzyme of GAD is present principally as the smaller of two molecular forms of GAD in brain. J Neurosci 11:2725-2731, 1991

23. Martinez RCR, de Oliveira AR, Brandão ML: Conditioned and unconditioned fear organized in the periaqueductal gray are differentially sensitive to injections of muscimol into amygdaloid nuclei. Neurobiol Learn Mem 85:58-65, 2006

24. Martinez RCR, Ribeiro de Oliveira A, Brandão ML: Serotonergic mechanisms in the basolateral amygdala differentially regulate the conditioned and unconditioned fear organized in the periaqueductal gray. Eur Neuropsychopharmacol 17:717-724, 2007

25. Martins MA, Carobrez AP, Tonussi CR: Activation of dorsal periaqueductal gray by glycine produces long lasting hyponociception in rats without overt defensive behaviors. Life Sci 83:118-121, 2008

26. Martins MA, De Castro Bastos L, Melo NEB, Tonussi CR: Dependency of nociception facilitation or inhibition after periaqueductal gray matter stimulation on the context. Behav Brain Res 214:260-267, 2010

27. Mota-Ortiz SR, Sukikara MH, Felicio LF, Canteras NS: Afferent connections to the rostrolateral part of the periaqueductal gray: a critical region influencing the motivation drive to hunt and forage. Neural Plast 2009:612698, 2009

28. Nichols DS, Thorn BE: Stimulation-produced analgesia and its cross-tolerance between dorsal and ventral PAG loci. Pain 41:347-352, 1990

29. Pagano RL, Assis DV, Clara JA, Alves AS, Dale CS, Teixeira
MJ, et al: Transdural motor cortex stimulation reverses neuropathic pain in rats: a profile of neuronal activation. Eur J Pain 15:268.e1-268.e14, 2011

30. Pagano RL, Fonoff ET, Dale CS, Ballester G, Teixeira MJ, Britto LRG: Motor cortex stimulation inhibits thalamic sensory neurons and enhances activity of PAG neurons: possible pathways for antinociception. Pain 153:2359-2369, 2012

31. Palazzo E, Guida F, Migliozzi A, Gatta L, Marabese I, Luongo L, et al: Intraperiaqueductal gray glycine and D-serine exert dual effects on rostral ventromedial medulla $\mathrm{ON}$ - and OFF-cell activity and thermoceptive threshold in the rat. $\mathbf{J}$ Neurophysiol 102:3169-3179, 2009

32. Paxinos G, Watson C: The Rat Brain in Stereotaxic Coordinates, ed 4. San Diego: Academic Press, 1998

33. Peng YB, Lin Q, Willis WD: Effects of GABA and glycine receptor antagonists on the activity and PAG-induced inhibition of rat dorsal horn neurons. Brain Res 736:189-201, 1996

34. Renno WM, Alkhalaf M, Mousa A, Kanaan RA: A comparative study of excitatory and inhibitory amino acids in three different brainstem nuclei. Neurochem Res 33:150-159, 2008

35. Sherman AD, Gebhart GF: Pain-induced alteration of glutamate in periaqueductal central gray and its reversal by morphine. Life Sci 15:1781-1789, 1974

36. Taylor CP: Mechanisms of action of gabapentin. Rev Neurol (Paris) 153 (Suppl 1):S39-S45, 1997

37. Timmerman $\mathrm{W}$, Westerink BH: Brain microdialysis of GABA and glutamate: what does it signify? Synapse 27:242-261, 1997

38. Tsubokawa T, Katayama Y, Yamamoto T, Hirayama T, Koyama S: Chronic motor cortex stimulation for the treatment of central pain. Acta Neurochir Suppl (Wien) 52:137-139, 1991

39. Tůma P, Šustková-Fišerová M, Opekar F, Pavlíček V, Málková K: Large-volume sample stacking for in vivo monitoring of trace levels of $\gamma$-aminobutyric acid, glycine and glutamate in microdialysates of periaqueductal gray matter by capillary electrophoresis with contactless conductivity detection. J Chromatogr A 1303:94-99, 2013

40. Vadalouca A, Siafaka I, Argyra E, Vrachnou E, Moka E: Therapeutic management of chronic neuropathic pain: an examination of pharmacologic treatment. Ann N Y Acad Sci 1088:164-186, 2006

41. Viisanen H, Pertovaara A: Antinociception by motor cortex stimulation in the neuropathic rat: does the locus coeruleus play a role? Exp Brain Res 201:283-296, 2010

42. Waagepetersen HS, Sonnewald U, Gegelashvili G, Larsson OM, Schousboe A: Metabolic distinction between vesicular and cytosolic GABA in cultured GABAergic neurons using $13 \mathrm{C}$ magnetic resonance spectroscopy. J Neurosci Res 63:347-355, 2001

43. Walker JM, Huang SM, Strangman NM, Tsou K, SañudoPeña MC: Pain modulation by release of the endogenous cannabinoid anandamide. Proc Natl Acad Sci U S A 96:1219812203, 1999

44. Watson AH: Antibodies against GABA and glutamate label neurons with morphologically distinct synaptic vesicles in the locust central nervous system. Neuroscience 26:33-44, 1988

45. Zhang X, Liu L, Zhang X, Ma K, Rao Y, Zhao Q, et al: Analytical methods for brain targeted delivery system in vivo: perspectives on imaging modalities and microdialysis. J Pharm Biomed Anal 59:1-12, 2012

46. Zimmermann M: Ethical guidelines for investigations of experimental pain in conscious animals. Pain 16:109-110, 1983

\section{Disclosures}

The authors report no conflict of interest concerning the materi- 
als or methods used in this study or the findings specified in this paper.

\section{Author Contributions}

Conception and design: Martinez, de Andrade, Pagano, Lopes, Fonoff. Acquisition of data: Martinez, de Andrade, Lopes, Auada, Gouveia, Antunes, Assis, Lebrun. Analysis and interpretation of data: Martinez, de Andrade, Pagano, Lopes, Auada, Lebrun, Fonoff. Drafting the article: Martinez, de Andrade, Pagano,

Fonoff. Critically revising the article: Martinez, Pagano, Auada, Gouveia, Antunes, Assis, Lebrun, Fonoff. Reviewed submitted version of manuscript: all authors. Approved the final version of the manuscript on behalf of all authors: Martinez. Statistical analysis: Martinez, de Andrade, Lebrun, Fonoff. Administrative/ technical/material support: Martinez, Pagano. Study supervision: Martinez, Fonoff.

\section{Supplemental Information}

Online-Only Content

Supplemental material is available with the online version of the article.

Supplemental Material. https://thejns.org/doi/suppl/10.3171/ 2018.7.JNS173239.

\section{Previous Presentations}

This work was presented and was awarded as the best oral presentation at the 2016 meeting of the ASSFN (American Society for Stereotactic and Functional Neurosurgery), June 18-21, Chicago, Illinois.

\section{Correspondence}

Raquel C. R. Martinez: Instituto de Ensino e Pesquisa, Hospital Sírio-Libanês, São Paulo, Brazil.quelmartinez@yahoo.com.br. 